\title{
Inversión en Tecnologías de la Información y las Comunicaciones y su relación con en el direccionamiento estratégico de las PYMES de Santiago de Cali - Colombia
}

\author{
Sandra Cristina Riascos $\mathrm{Erazo}^{1}$, Adriana Aguilera Castro ${ }^{1}$, Harold Armando Achicanoy ${ }^{2}$ \\ \{Sandra.riascos, Adriana.aguilera\}@correounivalle.edu.co,h.a.achicanoy@cgiar.org \\ ${ }^{1}$ Universidad del Valle - Facultad de Ciencias de la Administración, Calle 4B \# 36 - oo Ed. 124 Oficina 3001, \\ AA 25360, Santiago de Cali - Valle del Cauca, Colombia \\ ${ }^{2}$ Centro de Agricultura Tropical, Km 17, Recta Cali-Palmira, Valle del Cauca, Colombia
}

DOI: 10.17013/risti.18.1-17

\begin{abstract}
Resumen: Las organizaciones modernas han estimado como inversión prioritaria acceder a las Tecnologías dela Información y las Comunicaciones (TIC), con el principal propósito de obtener información confiable y oportuna que les permita establecer un direccionamiento adecuado con el contexto innovador y competitivo del siglo XXI. Este articulo pretende ilustrar la relación entre la inversión en TIC (Hardware, Software y Telecomunicaciones) y el direccionamiento estratégico en 107 PYMES de Santiago de Cali - Colombia, utilizando herramientas estadísticas como Test de Independencia Chi -cuadrado, imputación múltiple de datos y PLS-PM (Mínimos cuadrados parciales - Modelación de rutas). El principal resultado de la investigación, es que refleja que la inversión en TIC, específicamente en software, es uno de los principales apoyos para el direccionamiento estratégico de las organizaciones.
\end{abstract}

Palabras Clave: Inversión en Tecnología; TIC; Direccionamiento estratégico; PYMES.

Investment in Information Technology and Communications and its relationship with the strategic direction for SMEs in Cali-Colombia

\begin{abstract}
Modern organizations have given great importance to the investment in Communications and Information Technology (CIT), in order to obtain reliable and suitable information that enables them to establish an appropriate Strategic Management approach, according to the innovative and competitive context of the $21^{\text {st }}$ century. The aim of this paper is to illustrate the relationship between the investment in CIT (Hardware, Software and Telecommunications) and the Strategic Management Scheme among 107 SMEs in Cali - Colombia, by using statistical tools such as the Chi-Squared Test of Independence, the Multiple Imputation Data Technique, and the PLS-PM Methog (Partial Least Squares - Path Modeling). The most important outcome of this research work is finding that CIT investment, more precisely, Software Investment, is one of the most important factors impacting Strategic Management within organizations.
\end{abstract}

Keywords: Technology Investment; ICT, Strategic Management, SMEs. 


\section{Introducción}

La inversión en Tecnologías de la Información y las Comunicaciones (TIC) se constituye en una oportunidad para las organizaciones que pretenden mantenerse en el mercado como lo afirman Drucker (1970), Porter (1984), Weill (1992), Valor (2006), Aguilera y Riascos (2009), Taylor (2015), entre otros autores.

En The Global Information Technology Report (2015) se ilustra que Colombia se encuentra en el puesto 64 de 143 países a nivel mundial, con una calificación de 4,1 a nivel de inversión y desarrollo tecnológico, siendo 7 la máxima calificación. Este ranking se realiza considerando el impacto económico y social que genera la inversión en TIC; el impacto económico se evidencia por el número de solicitudes de patentes, así como por el papel de las TIC en el desarrollo de nuevos productos, procesos y modelos de organización. El impacto social que generan las TIC en un país, se refleja en el Producto Interno Bruto (PIB) como lo menciona la Unión Internacional de Telecomunicaciones (ITU, 2012); un aumento del 10\% en la penetración de internet de banda ancha, se podría traducir en un incremento de la tasa de crecimiento del PIB entre 0,25\% y 1,38\%. Según el Consejo Privado de Competitividad (2012), en Colombia, la inversión en TIC creció 162\% entre 2004 (4,6\% del PIB) y 2010 (5,4\% del PIB), impulsada principalmente por la categoría de comunicaciones, que compone el $87 \%$ de este sector; según esta entidad, la inversión en TIC ha aumentado considerablemente entre los años 2004 y 2010; el mayor porcentaje de inversión se realiza en Comunicaciones (180,3\%), le sigue Servicios (97,1\%), posteriormente software (75,44\%) y finalmente Hardware (75,3\%).

Las Pequeñas y Medianas empresas colombianas han encontrado en las TIC no solamente la posibilidad de optimizar sus procesos y actividades de negocio, sino que también pueden incorporar estas tecnologías como una estrategia corporativa que les permita fortalecerse para impulsar la economía del país. Es de anotar que el censo general de 2005 (DANE. 2005), revela que en Colombia las microempresas conforman el 96,4\% de los establecimientos; las pequeñas empresas el 3,0\%; las medianas el o,5\%; y las grandes empresas el o,1\%. Por personal ocupado, las microempresas representan el 50,3\% del empleo; las pequeñas el 17,6\%; las medianas el 12,9\%; y las grandes el 19,2\%; situación que se replica a nivel mundial según Muñoz, Gasca y Valtierra (2014); el claro aporte de las pymes al desarrollo económico de un País, fue la principal motivación para la realización de este trabajo. Considerando estudios que se han desarrollado para identificar el impacto de las TIC en las organizaciones de manera general (Sánchez, et al., 2014; Sagi et al., 2004; Ross y Blumenstein, 2013; Taylor, 2015); la investigación que dio origen a este trabajo, pretendió analizar de forma específica cual es la relación que existe entre la inversión en TIC y el direccionamiento estratégico de las PYMES de la ciudad de Santiago de Cali - Colombia; se utilizó el enfoque metodológico cuantitativo para lograr el objetivo de la investigación. En la primera parte del artículo se presenta el referente teórico, las tecnologías de la información y las comunicaciones y el direccionamiento estratégico; luego el modelo teórico, aquí se definen las variables; a continuación la metodología y luego los resultados y las conclusiones.

\section{Referente Teórico}

\subsection{Tecnologías de la Información y las Comunicaciones}

Las TIC hacen referencia a la informática y los dispositivos de comunicaciones, en esencia a gestionar la información (IDC, 2007); según Quiroga et al. (2010) las TIC 
son consideradas tecnologías digitales transversales, porque son aplicables a infinidad de procesos.

Las TIC se traducen en tres componentes fundamentales para que funcione de forma apropiada la gestión de la información, estos componentes según Amaya (200) son: Hardware: hace referencia a la infraestructura tecnológica que soporta los sistemas de información que se encuentran implementados en las organizaciones. El sistema de computación actual consiste en una unidad de procesamiento central, almacenamiento primario, almacenamiento secundario, dispositivos de entrada, dispositivos de salida y dispositivos de comunicación; Software: consiste en las instrucciones detalladas que controlan el funcionamiento de un sistema computacional, las principales funciones que tiene el software son: Administrar los recursos de Hardware, proporcionar las herramientas para aprovechar dichos recursos, actuar como intermediario entre las organizaciones y la información almacenada; Telecomunicaciones: se pueden definir como la comunicación de información por medios electrónicos, generalmente a cierta distancia; no únicamente en la transmisión de la voz por líneas telefónicas sino también la transmisión de datos a través de computadores de un lugar a otro. Las TIC confluyen en la interrelación de los aspectos de software, hardware y telecomunicaciones (figura 1), facilitando la generación de estrategias direccionadas al progreso de la organización.

\subsection{Inversión en TIC}

La Inversión en tecnología y específicamente en TIC se encuentra en un avance interesante como lo demuestran los reportes internacionales y nacionales (The Global Information Technology Report, 2015; Consejo Privado de Competitividad, 2015); esto permite establecer que las TIC son un factor importante y determinante para el crecimiento de las organizaciones. El papel que las TIC juegan en las empresas ha experimentado un cambio profundo, pasando de ser simples herramientas de tratamiento de datos a convertirse en la columna vertebral que afecta a todas las actividades de la organización, tanto a nivel interno como externo

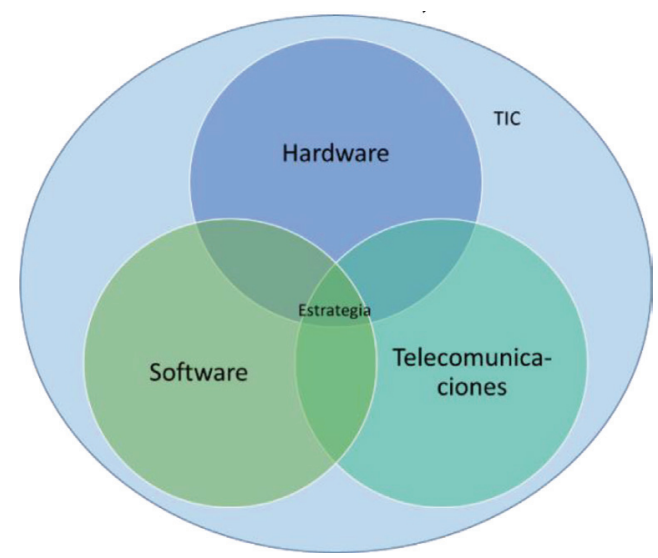

Figura 1 - Interrelación de Hardware, Software y Telecomunicaciones

Gómez y Suarez, 2011). Menshing y Adams (1998) citados por Maldonado, Mojica y Molina (2013) identifican que un incremento significativo en la inversión en TIC es 
uno de los factores más importantes que permiten un incremento significativo en la eficiencia de toda organización.

Jasso (2009) menciona que aunque en principio la inversión en TIC no evidencia rentabilidad, es conveniente seguir invirtiendo debido a que se constituye indirectamente, como elemento de sinergia, para dar mayor competitividad a la organización o porque es un elemento estratégico en la planeación organizacional. En el estudio empírico desarrollado por Bento et al (2014), en 1.990 negocios, se estableció a través del análisis estadístico de la información recolectada que las TIC tienen una relación directa con los resultados del negocio, especialmente en la eficiencia de las operaciones y el incremento de las ventas. Mihalic y Buhalis (2012) afirman en su estudio empírico del sector hotelero que las TIC complementan y potencializan otros recursos competitivos de la organización, justificándose de esta forma la inversión en este tipo de recursos.

\subsection{Direccionamiento estratégico}

Según Camacho (2002, p.2), el direccionamiento estratégico "es un enfoque gerencial que permite a la alta dirección determinar un rumbo claro y promover las actividades necesarias para que toda la organización trabaje en la misma dirección. Es importante destacar que cuando el autor habla de un rumbo claro, se refiere a planes establecidos de manera formal y que además sean reconocidos por todas las personas que trabajan en la organización.

Saloner, Shepard y Podolny (2005, p.1) plantean que "la administración estratégica tiene el objetivo fundamental de dar apoyo al administrador en la búsqueda continua de métodos mediante el desarrollo de un conjunto de herramientas y mapas conceptuales que permitan descubrir las relaciones sistémicas que existen entre las decisiones tomadas por el administrador y el desempeño alcanzado por la administración”. En este sentido, Aguilera y Riascos (2009) plantean que en las fases del direccionamiento estratégico pueden ser usadas herramientas TIC como apoyo a los procesos de toma de decisiones. Según las autoras, la fase de análisis estratégico genera gran cantidad de información que puede ser analizada empleando herramientas computacionales como 1) La diagramación de flujo de procesos, en este caso los software más utilizados son: el DFD, el Microsoft Visio, el Poseidon for UML y el Flow 5, entre otros; y 2) Software estadístico, que facilita el análisis de la información, como el SPSSo Statgraphics, Microsoft Excel y Open Office. Para la fase de formulación estratégica, Aguilera y Riascos (2009) proponen herramientas automatizadas, las TIC ofrecen técnicas como el Datamining, entre el software de apoyo para realizar este proceso se encuentra Matheo Analyzer y Tetralogie, también software estadístico como el SPSS. Otra herramienta que puede ser usada en esta fase, son los Árboles de decisión, el software que puede ser usado en este caso es GATree y DTreg. Además de lo anterior, los sistemas integrados, como los ERP, permiten obtener mayores beneficios para las organizaciones, entre los más usados se encuentran SAP, OpenERP y Oracle. En la fase de implantación estratégica, Aguilera y Riascos (2009) mencionan que las TIC ofrecen alternativas de software como Strategy Map Balanced Scorecard, la suite LABSAG (para simulación), RISK, y para los procesos de control COBIT (Control Objectives for Information an related Technology) y COSO (Committee of Sponsoring Organizations - of the Treadway Commission).

Estudios desarrollados en torno a la temática de las TIC y el direccionamiento estratégico destacan que la tecnología no sólo son herramientas operativas sino estratégicas 
en la construcción de escenarios direccionados a la competitividad y a la innovación (Baden-Fuller y Haefliger (2013); Muñoz, Gasca y Valtierra (2014); Aguilera y Riascos (2009)); de ahí la importancia de que la empresas realicen inversión en TIC. En la figura 2 se muestra la relación entre la inversión en TIC y el direccionamiento estratégico.

\section{Modelo Teórico}

El referente teórico expuesto y la pregunta de investigación planteada, permitieron a los investigadores estimar las variables utilizadas para el presente estudio; adicionalmente, el análisis realizado a las encuestas sobre tecnología, desarrolladas por PricewaterHouse Coopers (2013); Deloitte (2012); y FAEDPYME (2011), permitió identificar aspectos relacionados con tecnología y estrategia; las variables utilizadas en el estudio se relacionan en la tabla 1.

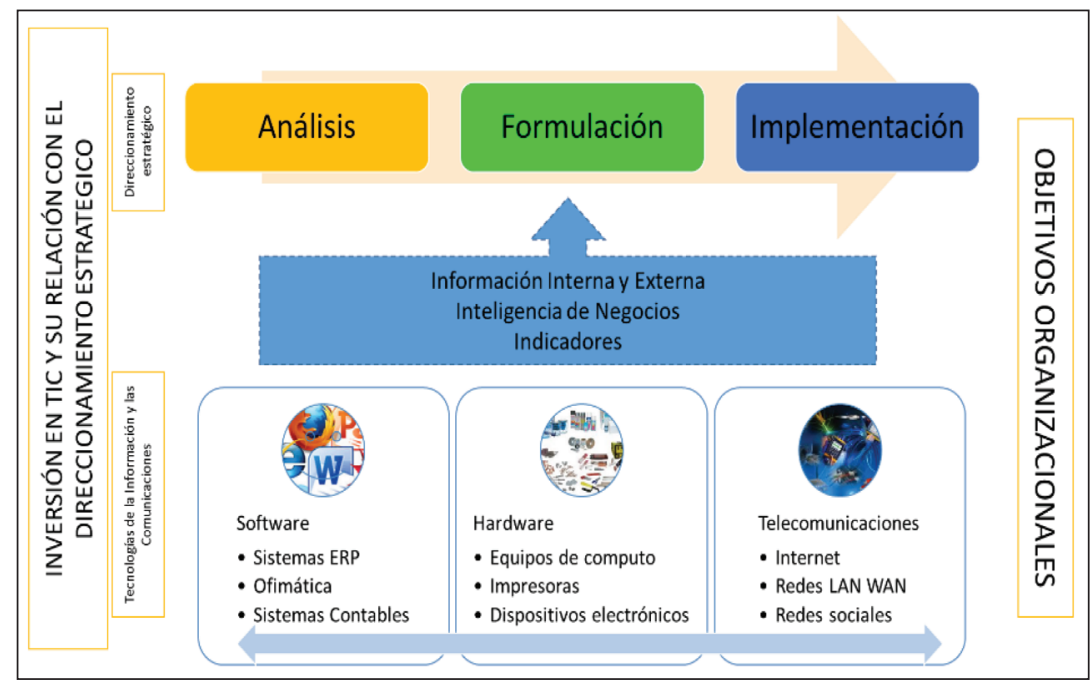

Figura 2 - Inversión en TIC y su relación con el direccionamiento estratégico

\begin{tabular}{llll}
\hline & Variables Independientes del Estudio & \multicolumn{1}{c}{$\begin{array}{c}\text { Variable Dependiente del } \\
\text { Estudio }\end{array}$} \\
\hline Hardware & \multicolumn{1}{c}{ Software } & Telecomunicaciones & $\begin{array}{c}\text { Direccionamiento } \\
\text { Estratégico }\end{array}$ \\
\hline $\begin{array}{l}\text { Clases de } \\
\text { Impresoras } \\
\text { (P.8) }\end{array}$ & $\begin{array}{l}\text { Sistemas de } \\
\text { Información para } \\
\text { desarrollo de nuevos } \\
\text { o mejores productos } \\
\text { (P.17.6) }\end{array}$ & $\begin{array}{l}\text { Conexión a Internet por Red } \\
\text { de datos (P.34.5) }\end{array}$ & $\begin{array}{l}\text { Los equipos de cómputo que } \\
\text { apoyan el direccionamiento } \\
\text { (P.6.9) }\end{array}$ \\
\hline $\begin{array}{l}\text { Dispositivos } \\
\text { para el ingreso } \\
\text { de datos (P. 9) }\end{array}$ & $\begin{array}{l}\text { Renovación de SI } \\
\text { (P.17.18) }\end{array}$ & $\begin{array}{l}\text { Servicios de comunicación } \\
\text { (P.36) }\end{array}$ & $\begin{array}{l}\text { Los Sistemas de Información } \\
\text { (SI) y software en general } \\
\text { apoyan la toma de decisiones } \\
\text { y estrategias de la empresa } \\
\text { (P.17.4) }\end{array}$ \\
\hline
\end{tabular}


Variables Independientes del Estudio

\begin{tabular}{|c|c|c|c|}
\hline Hardware & Software & Telecomunicaciones & $\begin{array}{l}\text { Direccionamiento } \\
\text { Estratégico }\end{array}$ \\
\hline $\begin{array}{l}\text { Dispositivos } \\
\text { biométricos } \\
\text { (P.10) }\end{array}$ & $\begin{array}{l}\text { Software ofimático y/o } \\
\text { Online (P.19) }\end{array}$ & $\begin{array}{l}\text { Tipo de conexión a Internet } \\
\text { (P.38) }\end{array}$ & $\begin{array}{l}\text { El uso de internet apoya la } \\
\text { generación de las estrategias } \\
\text { (P.34.9) }\end{array}$ \\
\hline $\begin{array}{l}\text { Equipos } \\
\text { audiovisuales } \\
\text { (P.11) }\end{array}$ & $\begin{array}{l}\text { SI ERP ((Enterprise } \\
\text { Resource Planning) } \\
\text { (P.47.2) }\end{array}$ & Tipo de red de datos (P.39) & \\
\hline \multirow[t]{4}{*}{$\begin{array}{l}\text { Equipos de } \\
\text { cómputo (P.18) }\end{array}$} & $\begin{array}{l}\text { SI CRM (Customer } \\
\text { Relationship } \\
\text { Management) P. (47.3) }\end{array}$ & $\begin{array}{l}\text { Uso de los Servicios de } \\
\text { Internet por los empleados } \\
\text { (P.41) }\end{array}$ & \\
\hline & $\begin{array}{l}\text { SI SCM (Supply Chain } \\
\text { Management) (P. 47.4) }\end{array}$ & $\begin{array}{l}\text { Uso de las Redes sociales } \\
\text { (P.45) }\end{array}$ & \\
\hline & & $\begin{array}{l}\text { Uso de los Servicios de } \\
\text { Internet por la empresa (P.46) }\end{array}$ & \\
\hline & & $\begin{array}{l}\text { Implementación de Comercio } \\
\text { electrónico (P.47.1) }\end{array}$ & \\
\hline
\end{tabular}

Tabla 1 - Variables del modelo
Variable Dependiente del Estudio

ccionamiento

El uso de internet apoya la generación de las estrategias (P.34.9)

El modelo conceptual que representa el análisis teórico realizado se ilustra en la figura 3, se pretende establecer la relación entre la inversión en TIC (Hardware, Software y Telecomunicaciones) y el direccionamiento estratégico de las PYMES.

Para el estudio sólo se tomaron los aspectos más representativos de las variables independientes y que están siendo usados actualmente por las pymes. Las variables independientes (Hardware, Software y Telecomunicaciones) aportan al direccionamiento estratégico entregándole información de calidad, es decir, se encargan de desarrollar la

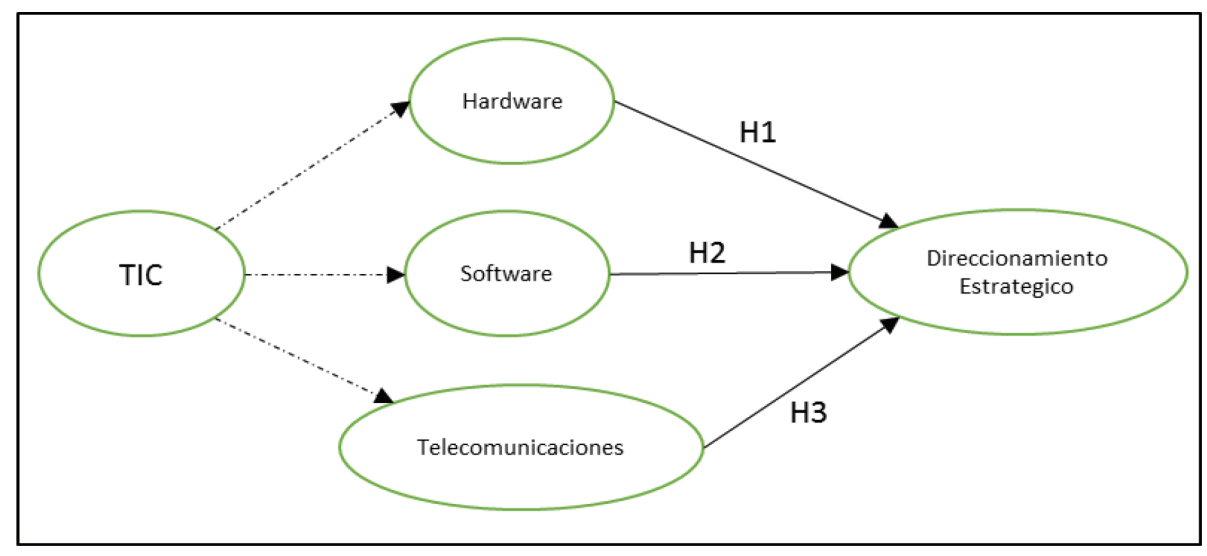

Figura 3 - Modelo Teórico 
transformación de datos a información útil para el proceso de definición de estrategias para la organización, esto se evidencia en: Hardware (dispositivos biométricos, lectores de códigos de barras, clases de impresoras, equipos de cómputo) a través del cual facilita el ingreso de datos y salida de información; Software (CRM, SCM, ERP, Ofimática, Sistemas contables) que facilitan el procesamiento de los datos generando información relevante para la organización; Telecomunicaciones (email, red de datos, redes sociales, servicios de internet, comercio electrónico) permiten el transporte y comunicación de la información del core del negocio a las diferentes áreas de la organización que requieren generar estrategias.

\section{Metodología}

El estudio se desarrolló conservando los rasgos del método científico dado que su punto de partida es, en general, una teoría previa o un conjunto racional y sistemático de ideas sobre la realidad que se trata (Sierra, 2003); además responde a las características del tipo de investigación cuantitativa, donde se establecen hipótesis que se comprueban mediante la utilización de herramientas estadísticas. La investigación se establece como descriptiva - correlacional, considerando que los estudios descriptivos por lo general fundamentan las investigaciones correlaciónales (Hernández, Fernández, Baptista, 1994). Los estudios descriptivos buscan especificar las propiedades importantes de personas, grupos, comunidades o cualquier otro fenómeno que sea sometido a análisis y los estudios correlaciónales permiten saber cómo se puede comportar un concepto o variable conociendo el comportamiento de otra u otras variables relacionadas (Dankhe (1976)).

El estudio se desarrolló con la información de 107 empresas PYME de los sectores primario, secundario y terciario de la ciudad de Santiago de Cali (Colombia); a las que se aplicó un cuestionario estructurado con preguntas en escala de Likert para facilitar el análisis e interpretación de la información obtenida. La recolección de la información fue realizada a través de correo electrónico y llamadas telefónicas, únicamente a los gerentes de las PYMES involucradas en el estudio. El análisis de la información se realizó teniendo en cuenta las siguientes cinco etapas, se esperaba que los resultados obtenidos en las etapas 2 y 4 no variaran significativamente, es decir, conservaran la estructura y las relaciones entre las variables estudiadas.

Etapa 1. Organización de datos: Codificación de variables de acuerdo a su escala de medición: para variables categóricas utilizar las escalas, nominal y ordinal; para variables con opción de respuesta múltiple, generar tantas variables nominales como opciones de respuesta se disponga. Para todo el conjunto de variables en la base de datos se omitieron las categorías asociadas a mediciones negativas o ambiguas. Por otrolado, seañadióla restricción deconservar variables con un porcentaje de datos faltantes inferior al 15\% (en términos absolutos, 16 datos faltantes como máximo por variable). Esto con el fin de realizar la posterior imputación de los mismos sin afectar la calidad de la información imputada y la estructura de la matriz de datos. Finalmente, se eliminó del estudio la información correspondiente a una empresa, ya que no cuenta con red de datos, lo que implica que la sección de Telecomunicaciones en el cuestionario carece de todas las respuestas para las variables asociadas; por tanto, la muestra definitiva queda conformada por 106 PYMES de la ciudad de Santiago de Cali - Colombia.

Etapa 2. Análisis de información sin tratamiento: especificación de constructos a analizar. Para el estudio se definieron a priori los constructos o variables latentes 
(medidas a partir de ítems del cuestionario estructurado) y su papel en el modelo estructural como se ilustran en la tabla 2.

\begin{tabular}{ll}
\hline Constructo & Papel a desempeñar \\
\hline Inversión en Hardware & Variables predictoras \\
\hline Inversión en Software & Variables predictoras \\
\hline Inversión en Telecomunicaciones & Variables predictoras \\
\hline Direccionamiento estratégico & Variables de respuesta \\
\hline
\end{tabular}

Tabla 2 - Variables a estudiar y su papel en el modelo estructural

Dado que el interés del estudio radica en la exploración de relaciones entre los constructos definidos, como primera medida se examinaron las relaciones entre todos los posibles pares de variables mediante Test de Independencia Chi-cuadrado tomando como nivel de significancia de la prueba, $\alpha=5 \%$.

Etapa 3. Análisis e imputación de datos faltantes: se verificó la proporción de datos faltantes por variable (imponiendo una restricción como máximo de 15\%) y por número de casos, con el propósito de determinar la influencia que se obtendría con la imputación de dicha información faltante. Esta fase se desarrolló mediante procedimientos de imputación múltiple, es decir, a partir de la matriz original se generan M matrices completas. Específicamente, se hizo uso de la técnica de imputación múltiple de datos: Multivariate imputation by chained equations (Van Buuren \& Groothuis-Oudshoorn, 2011). Aquí se definió un número de imputaciones múltiples $\mathrm{M}=50$. No obstante, para simplificar el número de análisis posteriores se realizó una variación del método de Rubin (Rubin, 1987), reduciendo a priori el conjunto de 50 matrices de datos con los valores imputados a una sola, asignando para cada caso donde se presenta un dato faltante el valor máximo de las categorías estimadas en el proceso de imputación.

Etapa 4. Análisis de información imputada: para comparar los resultados obtenidos con la información original e imputada de la matriz de asociación, se efectuó un Test de Mantel para analizar la correlación entre las matrices de asociación usando el coeficiente de correlación no paramétrico de Spearman. Posterior a esto, se realizó un proceso de cuantificación óptima de la matriz de datos imputados mediante un Análisis de Componentes Principales no Lineal (Meulman et al., 2004) esto con el propósito de cuantificar las categorías de respuesta de las variables categóricas, lo cual facilitó en los posteriores análisis la utilización de técnicas para variables cuantitativas. Finalmente, se realizó la aplicación del Análisis de Componentes Principales (PCA), para las variables que definen cada uno de los constructos con el fin de identificar asociaciones de interés que permitan refinar la definición de cada constructo.

Etapa 5. Ajuste y evaluación del modelo PLS-PM: Partial LeastSquares-Path Modeling PLS-PM a través de esta técnica estadística de modelación se buscó tratar las relaciones entre bloques de variables a través de métodos de regresión, permitiendo 
explorar la asociación entre variables latentes (o no observables) que son calculadas a partir de ítems cuantificables (Sanchez, 2013). El modelo consta de dos partes:

- Modelo estructural o modelo interno, es la parte del modelo que explora las relaciones entre variables latentes.

$$
L V_{j}=\beta_{0}+\sum_{i \rightarrow j} \beta_{j i} L V_{i}+\text { error }_{j}
$$

Donde,

$L V_{j}$ : Variable latente de interés o respuesta; $L V_{i}:$ Variables latentes predictoras.

$\beta_{j i}$ : Coeficientes de regresión que cuantifican el aporte de cada una de las variables latentes predictoras a la variable de respuesta; error $_{j}$ : Error aleatorio que cuantifica la variabilidad no explicada por las variables latentes incluidas en el modelo.

- Modelo de medida o modelo externo, corresponde a la parte del modelo que cuantifica las relaciones entre cada variable latente con su propio bloque de variables medidas. En este punto se distinguen dos casos de especial interés: el primero denominado modelo reflectivo, donde la variable latente se considera una causa de las variables medidas. El otro caso corresponde al modelo formativo, en el cual las variables medidas se consideran como variables predictoras de la variable latente. Para el estudio, se consideró un modelo formativo, cuya expresión matemática se presenta a continuación:

$$
L V_{j}=\lambda_{0 j}+\bar{\lambda}_{j k} X_{j k}+\text { error }_{j}
$$

Donde,

$L V_{j}$ : Variable latente; $\lambda_{j k}$ : Coeficientes o cargas que describen el nivel de asociación entre cada ítem o variable medida y su correspondiente variable latente

$X_{j k}$ : Variable observada que se encuentra asociada a una variable latente de interés.

error $_{j}$ : Error aleatorio que mide la variación no explicada por las variables observables que se incluyeron en el modelo.

Estos pasos se realizaron mediante un procedimiento iterativo, en el cual de manera inicial se asignaron pesos que permiten dar una cuantificación a las variables latentes (variables no observables) y posterior a esto se aplican las regresiones presentadas, repitiendo este proceso hasta alcanzar un nivel deseado de convergencia. La evaluación del modelo PLS-PM inicia a través del modelo de medida o externo, en el cual dependiendo de su construcción (reflectivo o formativo), para el caso de un modelo formativo se examina el valor absoluto de las cargas estimadas y se prueba la posible existencia de multicolinealidad entre las variables medidas. Posteriormente, se continúa con la 
evaluación del modelo estructural donde se examinan los coeficientes de determinación y los coeficientes estimados mediante técnicas de remuestreo. La herramienta utilizada para las diferentes etapas de análisis de la información fue el software estadístico $\mathrm{R}$ versión versión 3.1.2, $\mathrm{R}$ core team (2014).

\section{Resultados}

La mayor parte de la muestra se encuentra constituida por empresas del sector terciario (57\%) o de servicios, cuyo número de empleados es inferior a 50. Además las principales actividades económicas que predominan en la muestra son el comercio (44.9\%) y las industrias de bienes de consumo (28\%).

Luego en el heatmap de la Figura 4 se presentan los resultados del Test de Independencia Chi-cuadrado, calculado para todos los posibles pares de variables; existen pequeños parches de color rojo que corresponden a valores-p relativamente bajos, lo que indica asociaciones significativas entre grupos de variables, en este sentido utilizando un nivel de significancia $\alpha=0.05$ se obtiene un porcentaje del $7.8 \%$ de asociaciones significativas entre todas las comparaciones por pares de variables, dando a entender que probablemente existen patrones de relación en los datos que es necesario explorar. Estos cálculos se realizan con la información original, es decir, sin realizar la imputación de valor faltante alguno.

Por otro lado, el número de datos faltantes se evaluó desde diferentes puntos de vista, por un lado se tiene que el porcentaje de datos faltantes por variables respecto al número total de las mismas equivale a un $18 \%$ de las variables; mientras la relación por casos alcanza un cuarto del total de filas de la matriz, en este sentido si se ajusta el modelo únicamente con las filas que contienen la totalidad de la información se está perdiendo un $25 \%$ de empresas que pueden aportar en la explicación de las relaciones teóricas planteadas (ver Figura 5).

Paso siguiente se realizó la exploración del patrón de datos faltantes como se exhibe en la Figura 6. Los espacios en blanco representan los vacíos de información en la matriz de datos. Como puede observarse la distribución de los valores faltantes en la matriz de información no parece presentar un patrón definido, por tanto, el proceso de imputación de datos faltantes se maneja desde un marco de referencia aleatorio. Para efectuar la imputación de los valores faltantes mediante el procedimiento Multivariate imputation by chained equations se construyeron un total de 50 matrices de datos a través de 5 iteraciones múltiples. Con este método se logra determinar la estabilidad de las imputaciones y se selecciona como matriz de análisis final el valor con mayor frecuencia por dato faltante (por cada dato faltante se obtienen múltiples valores imputados y se selecciona como valor definitivo la categoría con mayor frecuencia). Con la nueva matriz de datos incluyendo los valores imputados se obtuvo un porcentaje de asociaciones significativas del 8.0\% entre pares de variables (ver Figura 7).

Adicional a esto se estudió la similaridad de las matrices de asociación a través del Test de Mantel realizando 10.000 permutaciones para obtener una aproximación realista de la significancia del test (figura 8). 

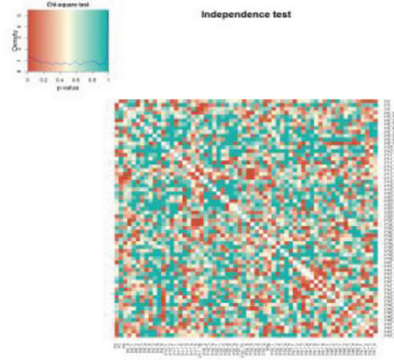

Figura 4 - Valores-p correspondientes al test de independencia Chi-cuadrado entre todos los posibles pares de variables, la diagonal superior e inferior contiene los mismos valores

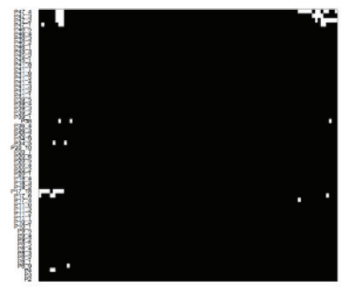

Figura 6 - Patrón de datos faltantes en la matriz de datos

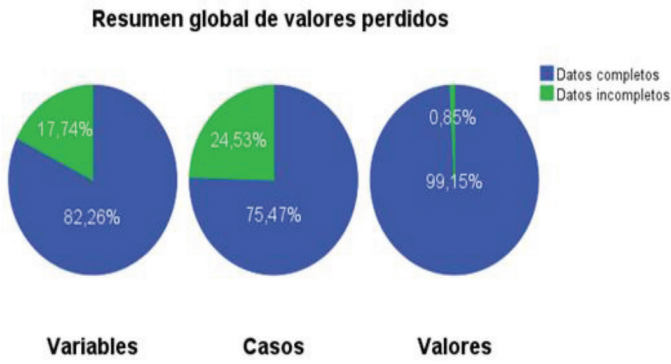

Figura 5 - Porcentaje de datos faltantes desde diferentes perspectivas

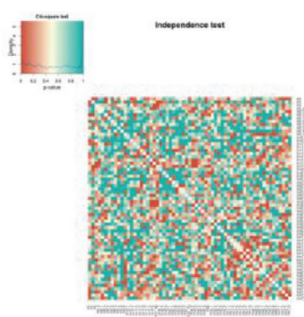

Figura 7 - Valores-p correspondientes al test de independencia Chi-cuadrado entre todos los posibles pares de variables sobre la matriz de datos imputados

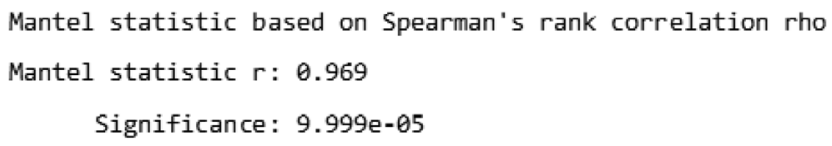

Figura 8 - Test de Mantel

Esto permite obtener una correlación entre las matrices de asociación de datos aproximada del 97\%, lo que indica una mínima perdida en la estructura de asociaciones entre las matrices de asociación de datos originales e imputados. Para estudiar la asociación entre variables p (descritas en la tabla 1) desde el punto de vista de cada uno de los constructos, se aplicó un PCA para los constructos que constituyeron las variables explicativas del modelo. En las Figuras 9, 10 y 11 se puede observar la fuerte asociación que existe en cada uno de los tres bloques de variables, formándose conglomerados de variables que tienden a medir constructos o variables latentes bien definidas. 
Los mapas factoriales ilustrados en las figuras 9, 10 y 11 evidencian que las preguntas del cuestionario asociadas con los dominios de Hardware, Software y Telecomunicaciones se encuentran fuertemente relacionadas con la primera componente principal. Esto significa que todas ellas contribuyen de manera apropiada en la explicación de los constructos asociados. Finalmente, en términos del ajuste del modelo obtenemos cargas positivas para todas las variables respecto a sus constructos, indicando así una correlación positiva entre cada una de las variables p (descritas en la tabla 1) que definen

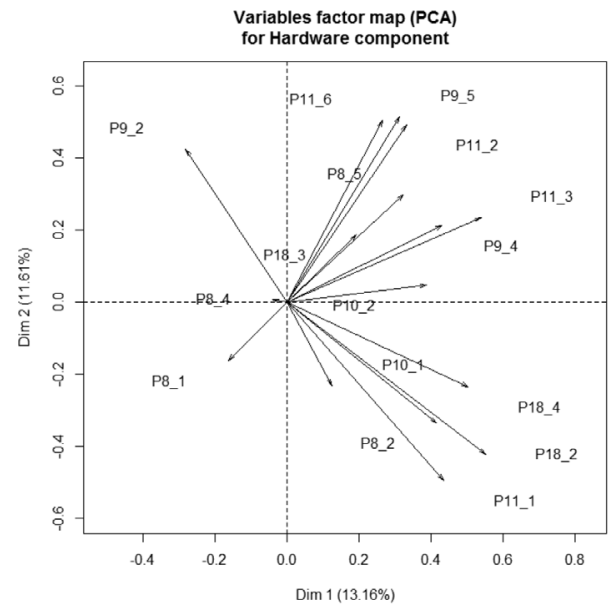

Figura 9 - Mapa factorial de variables, componente Hardware

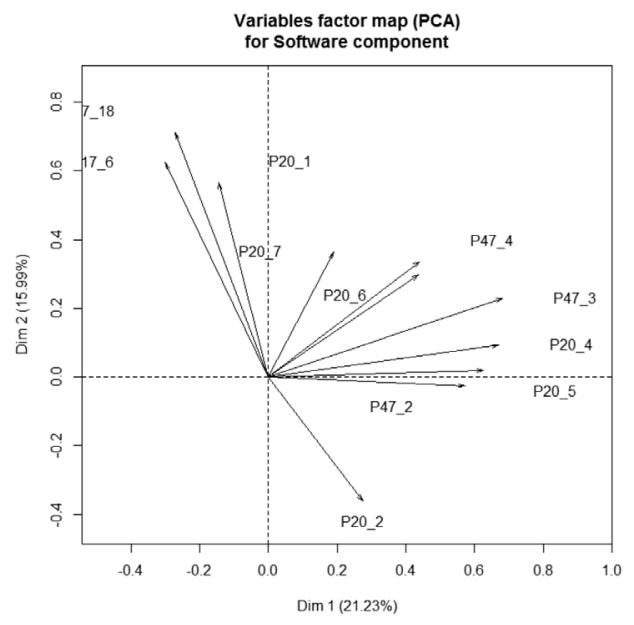

Figura 10 - Mapa factorial de variables, componente Software

la respectiva variable latente; excepto para dos variables del componente de inversión en hardware (ver Figura 12).

Además de analizar las cargas de las variables con su respectivo constructo también se presentan a continuación las cargas cruzadas, es decir la correlación que presenta cada una de las variables medidas con los demás constructos definidos, esto permite concluir de igual manera la adecuación de dichas variables para la explicación de las variables latentes (ver Figura 13). Es de anotar que las variables que definen cada uno de los constructos muestran los niveles más altos de asociación a diferencia de la asociación que se presenta entre estos y los demás constructos, lo que permite concluir la adecuación de las cargas y variables utilizadas. Evaluando el modelo estructural en la Figura 13, se observa el aporte positivo de la inversión en Software sobre el Direccionamiento Estratégico de las empresas estudiadas, este presenta una correlación del 68\% a diferencia de los componentes de inversión en Hardware y Telecomunicaciones, las cuales aunque presentan estimaciones negativas con base a los valores-p obtenidos se puede concluir que dichos parámetros del modelo no pueden descartarse al ser estadísticamente distintos de cero y por tanto tener un aporte bajo o nulo en la explicación de la variación asociada al Direccionamiento estratégico (Tabla 3). 


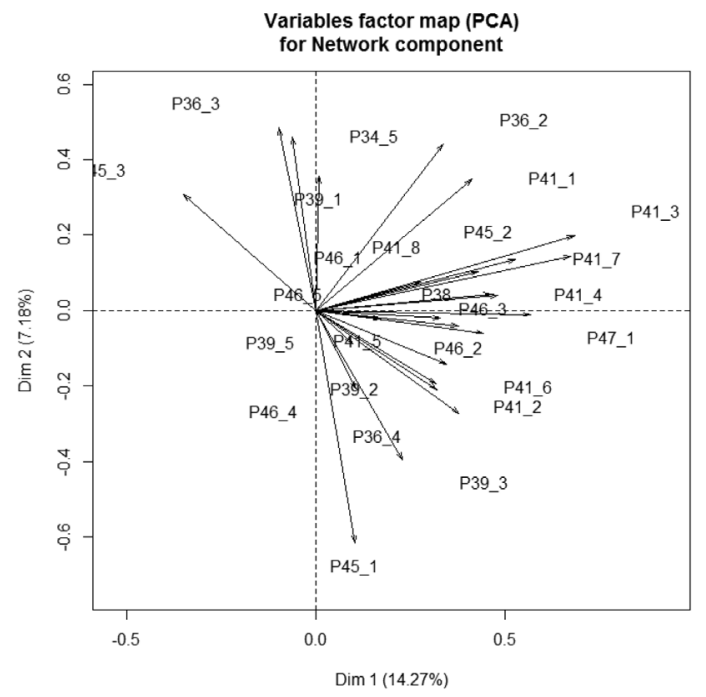

Figura 11 - Mapa factorial de variables, componente Telecomunicaciones
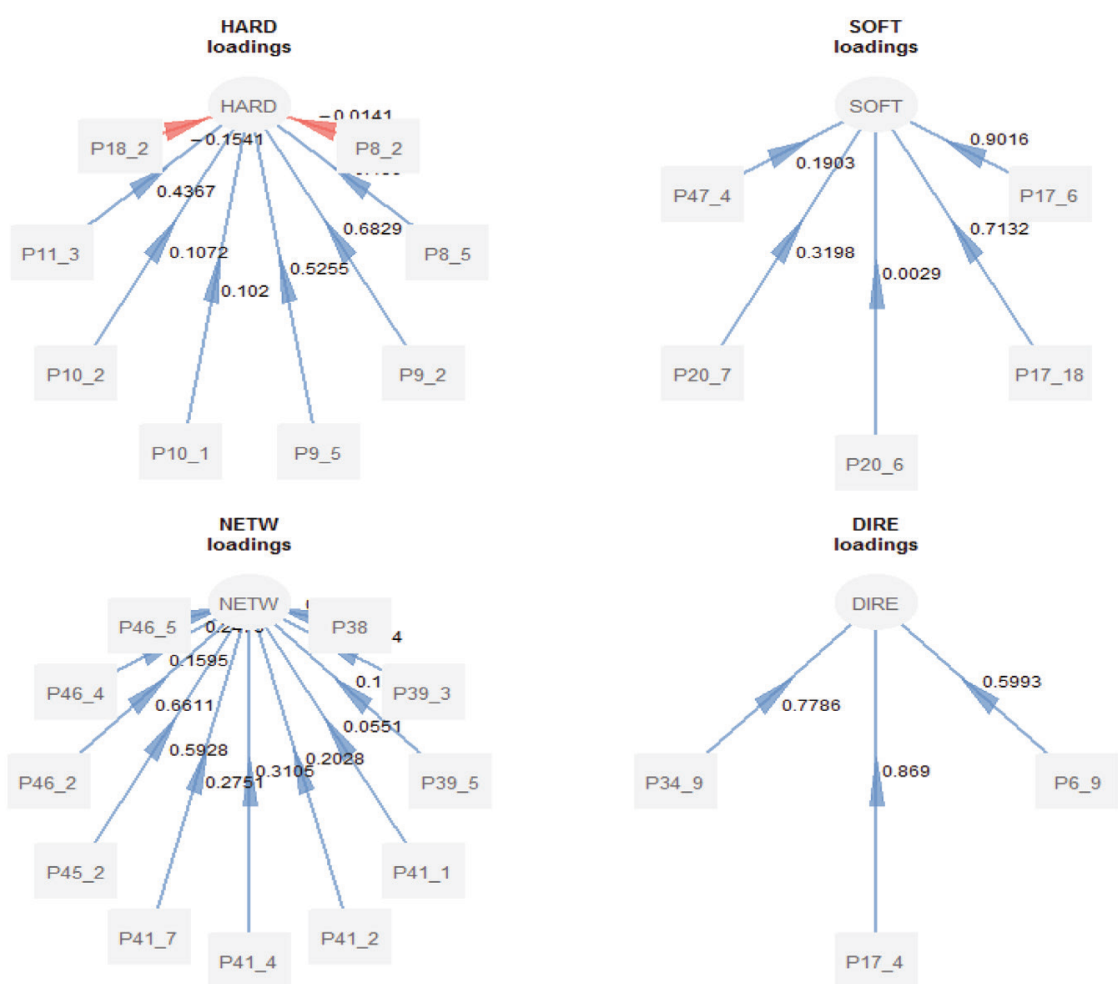

Figura 12 - Cargas factoriales de las variables observadas al constructo. 


\begin{tabular}{lllll}
\hline & Estimate & Std. Error & t-value & $p$-value \\
\hline Innovación en hardware & -0.086 & 0.069 & -1.252 & 0.213 \\
\hline Innovación en software & 0.683 & 0.069 & 9.905 & 0.000 \\
\hline Innovación en redes & -0.118 & 0.071 & -1.669 & 0.098 \\
\hline
\end{tabular}

Tabla 3 - Resumen de los parámetros del modelo

\section{Conclusiones}

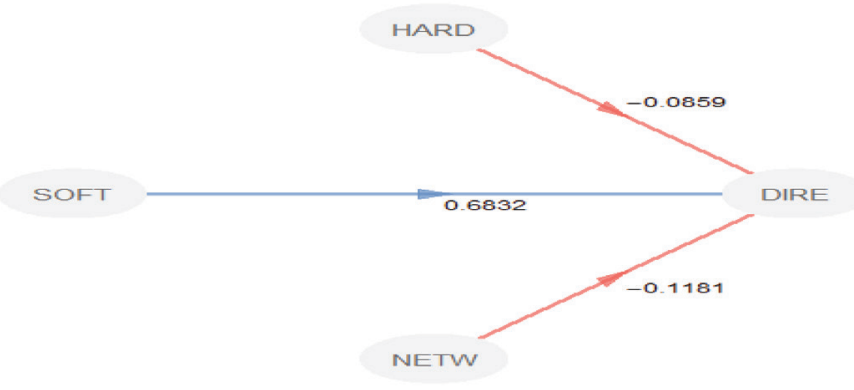

Figura 13 - Coeficientes del modelo estructural

En los estudios realizados a nivel de inversión en TIC se evidencia que las organizaciones le han otorgado un nivel de importancia estratégica a este aspecto. En las fases de Análisis, Formulación e Implementación, se requiere información interna y externa de la organización, aspectos de inteligencia de negocios e indicadores que pueden obtenerse a través de software, hardware y telecomunicaciones.

En los resultados de la investigación se comprueba que la inversión en Hardware no tiene relación directa con el direccionamiento estratégico de las Pymes de Santiago de Cali - Colombia; su bajo aporte al proceso de Direccionamiento Estratégico se puede explicar debido a que el hardware constituye el soporte tanto para el software como para las telecomunicaciones. En este sentido, para los empresarios, el hardware, por sí mismo no constituye un aporte importante al proceso. Por otra parte, el estudio confirma que la inversión en Software si tiene relación directa con el direccionamiento estratégico de las Pymes de Santiago de Cali - Colombia; esto debido a que se percibe el valor agregado por el procesamiento de los datos que generan información relevante para la toma de decisiones. Se invierte principalmente en sistemas de información contable, ofimática y ERP. Así mismo, la investigación demuestra que la inversión en Telecomunicaciones no tiene relación directa con el direccionamiento estratégico de las Pymes de Santiago de Cali - Colombia; esto debido principalmente a que las comunicaciones electrónicas aún no son completamente aceptadas por parte de los empresarios. El aporte de los 
beneficios que ofrece Internet no es percibido por los empresarios y por lo tanto no destinan recursos para su apropiación.

Limitaciones: las variables están en escala Likert, lo que impide obtener estadísticas descriptivas; además el alcance de la investigación no contemplo diferenciar los resultados por el sector económico ni por el tamaño de las empresas.

\section{Referencias Bibliográficas}

Aguilera, A. y Riascos, S. (2009). Direccionamiento Estratégico apoyado en las TIC. Revista Estudios Gerenciales. 25 (111), 127-143. doi:10.1016/So123-5923(09)70074-9

Baden-Fuller, C., \& Haefliger, S. (2013). Business models and technological innovation. Long Range Planning, 46(6), 419-426. doi:10.1016/j.lrp.2013.08.023

Bento, A., Bento, R., Ferreira White, L. (2014). Strategic Performance Management Systems: Impact on Business Results. Journal of Computer Information Systems, 54(3), 25-33.

Camacho M., (2002). Direccionamiento Estratégico: análisis de una herramienta poderosa. Revista vía Salud, 21, 6-12.

Consejo Privado de Competitividad (2012). Capitulo Tecnología de la Información y las Telecomunicaciones. Informe de Competitividad. Recuperado de: http://www. compite.com.co/site/informe-nacional-de-competitividad-2014-2015/

Consejo Privado de Competitividad (2015). Capitulo Tecnología de la Información y las Telecomunicaciones. Informe de Competitividad. Recuperado de: http://www. compite.com.co/site/informe-nacional-de-competitividad-2014-2015/

DANE (2005). Censo General 2005. Recuperado de: http://www.dane.gov.co/daneweb_ Vo9/index.php?option=com_content\&view=article\&id=307\&Itemid=124 .

Dankhe, G. L. (1976). Investigación y comunicación, en C. Fernández-Collado y G.L., Dankhe (Eds): La comunicación humana: ciencia social (pp. 385-454). México, México: McGraw Hill.

Deloitte (2012) Global Report 2012. Recuperado de: http://public.deloitte.com/ media/0564/index.html

Drucker, P. (1970). Tecnología, Administración y Sociedad. México, México: Roble.

FAEDPYME (2011). Análisis estratégico para el desarrollo de la Mipyme - Iberoamérica. Recuperado de: http://www.gaedpyme.upct.es/documentos/Faedpyme_ iberoamerica.pdf

Gómez, A. y Suarez, C. (2011) Sistemas de información: Herramientas prácticas para la gestión empresarial. México, México. RA-MA Editorial.

Hernández, Fernández y Baptista (1994). Metodología de la investigación, México, México. Mc Graw Hill. 
IDC (2007). TIC para PYMES. Madrid, España. LID Editorial.

ITU. (2012). Impact of Broadband on Economic Growth. Geneva: Telecommunication Development Bureau. Recuperado de: https://www.itu.int/ITU-D/treg/ broadband/ITU-BB-Reports_Impact-of-Broadband-on-the-Economy.pdf

Jasso, V. J. (2009). El valor de la tecnología en el siglo XXI. México, México: Universidad Nacional Autónoma de México.

Laudon K y Laudon J (2012). Sistemas de Información Gerencial. México, México. Pearson Educación.

Maldonado, G., Mojica, J. E., \& Molina, V. M. (2013). La relación entre la Innovación, las TICs y la calidad: Una Perspectiva de La Pyme Iberoamericana. Global Conference On Business \& Finance. Proceedings, 1135-1140.

Meulman, J. J., Van der Kooij, A. J., y Heiser, W. J. (2004). Principal components analysis with nonlinear optimal scaling transformations for ordinal and nominal data. Handbook of quantitative methodology for the social sciences, 49-70.

Mihalic, T., Buhalis, D. (2012). ICT As A Competitive Advantage Factor - Case Of Small Transitional Hotel Sector. Conference Proceedings: International Conference Of The Faculty Of Economics Sarajevo (ICES), 885-905.

Muñoz, M., Gasca. G, y Valtierra C. (2014). Caracterizando las Necesidades de las Pymes para Implementar Mejoras de Procesos Software: Una Comparativa entre la Teoría y la Realidad. RISTI - Revista Ibérica de Sistemas e Tecnologias de Informação. (E1), 1-15. DOI: 10.4304/risti.e1.1-15.

Oxford University Press. The Global Information Technology Report (2015). Recuperado de: http://reports.weforum.org/global-information-technology-report-2015/

Porter, M. (1984). Estrategia competitiva: Técnicas para el análisis de los sectores industriales y de la competencia. México, México: Continental.

PricewaterHouse Coopers (2013) Global Innovation Survey, Recuperado de: http://www.pwc.com/us/en/consulting-services.html

Quiroga, D., Quiroga, D., Vásquez, F., Montaño, H., Espinosa, D., Hernández, B., \& Gutiérrez, P. (2010). La gestión del conocimiento: y las tecnologías de la información y la comunicación en las organizaciones. Cali, Colombia: Universidad Autónoma de Occidente.

Ross, P., y Blumenstein, M. (2013). Cloud computing: the nexus of strategy and technology. Journal of Business Strategy, 34(4), 39-47. doi 10.1108/JBS-10-2012-0061

Rubin, D. B. (1987). Multiple Imputation for Nonresponse in Surveys. Harvard University, 15-19. doi: 10.1002/9780470316696

Sá, F., \& Rocha, Á. (2012). Definição da arquitetura de informação em organismo da administração pública local. RISTI-Revista Ibérica de Sistemas e Tecnologias de Informação, (10), 51-64. 
Sagi, J., Carayannis, E., Dasgupta, S., \& Thomas, G. (2004). ICT and Business in the New Economy: A Cross-Cultural Study of Attitudes towards eCommerce. Journal of Global Information Management, 12(3), 14-64.

Saloner, G., Shepard, A. \& Podolny, J. (2005). Administración estratégica. México, México: Limusa Wiley.

Sánchez, G. (2013). PLS path modeling with R. Trowchez Editions, Berkeley.

Sánchez, M., Palacios, B., López, C. y Sánchez, A. (2014). Percepciones de los empresarios de Pymes rurales sobre la integración de las TIC. RISTI. (E2). 71-84. doi: 10.17013/ risti.e2.71-84

Sierra, R (2003): Tesis doctorales y trabajos de investigación científica. Metodología general de su elaboración y documentación, Madrid, España: Paraninfo.

Taylor, P. (2015). The Importance of Information and Communication Technologies (ICTs): An Integration of the Extant Literature on ICT Adoption in Small and Medium Enterprises. International Journal of Economics, Commerce and Management, 3(5). 274-295.

Valor, J. (2006). Acertar en la tecnología. IESE, Revista de Antiguos alumnos, (100), 100-105.

Van Buuren, S., \& Groothuis-Oudshoorn, K. (2011). Multivariate Imputation by Chained Equations. Journal Of Statistical Software, 45(3), 1-67. doi: 10.1177/0962280206074463

Weill, P. (1992). The Relationship between Investment in Information Technology and Firm Performance: A Study of the Valve Manufacturing Sector. Information Systems Research, 3(4), 307-333. doi: 10.1287/isre.3.4.307 